Rev Inv Vet Perú 2019; 30(3): 1328-1335

http://dx.doi.org/10.15381/rivep.v30i3.16593

\title{
Comunicación
}

\section{Estudio clínico-patológico del Sarcoide Fibroblástico Equino: reporte de caso clínico}

\author{
Clinical-pathological study of Equine Sarcoid Fibroblastic: clinical \\ case report
}

\author{
Juan Carlos Pinilla León ${ }^{1,2}$, Angel Alberto Florez ${ }^{1}$
}

\section{Resumen}

Se describe el caso en un equino Paso Fino de Colombia de 6 años que presenta una masa recidivante de aspecto irregular, granulomatosa, ulcerativa y hemorrágica en la región del prepucio y pene. Se practicó resolución quirúrgica del tumor, estudio histopatológico y tratamiento posoperatorio del equino. Las características macroscópicas y descripción histológica de las lesiones estudiadas ponen en evidencia la presencia de un Sarcoide de tipo fibroblástico.

Palabras clave: fibroblástico; sarcoide equino; tumor

\section{Abstract}

The case is described in a 6-year-old Colombian Paso Fino equine that presents a recurrent mass of irregular, granulomatous, ulcerative and hemorrhagic appearance in the prepuce and penis region. Surgical resolution of the tumor, histopathological study and postoperative treatment of the equine were performed. The macroscopic characteristics and histological description of the lesions studied showed the presence of a fibroblastic Sarcoid type.

Key words: fibroblastic; equine sarcoid; tumor

\footnotetext{
${ }^{1}$ Universidad de Santander, Facultad de Ciencias Exactas, Naturales y Agropecuarias, Grupo de Investigación en Ciencia Animal-GICA, Bucaramanga, Colombia

${ }^{2}$ E-mail: jcpinilla@hotmail.com
}

Recibido: 20 de octubre de 2018

Aceptado para publicación: 20 de abril de 2019 


\section{INTRODUCCIÓN}

Los sarcoides equinos son estructuras tumorales que representan entre el 30 y $90 \%$ de la casuística de tumores cutáneos en los équidos (Scott y Miller, 2003; Bergvall, 2013). Los tumores se ubican preferiblemente en la cabeza, abdomen ventral y miembros del animal (McMahlan y Dubovi, 2011); sin embargo, Sperotto (2010) reportó una mayor presentación en la comisura de los ojos, pecho, extremidades, tronco, abdomen, prepucio y cara. Se reporta en caballos, asnos y mulas, especialmente en animales jóvenes ( $<4$ años), afectando ambos sexos y sin distinción de raza o pelaje (McMahlan y Dubovi, 2011). El término de sarcoide se debe a la apariencia sarcomatosa maligna de las lesiones (Cremasco y Sequera, 2010).

Según Bergvall (2013), el sarcoide equino tiene una etiología multifactorial, incluyendo heridas, infecciones virales y predisposición genética (Jubb et al., 2007). Se ha propuesto la participación de un virus oncogénico de la familia Retroviridae; sin embargo, existen fuertes hallazgos que postulan al Papilomavirus (PVB) tipos 1 o 2 como los agentes etiológicos (Knottenbelt y Mattews, 2001; Yuan et al., 2007). Probablemente, genes específicos del sistema inmune sean regulados por el PVB en el equino (Finlay et al., 2009). Por otro lado, la presencia de sarcoide ha sido asociada con equinos que conviven en hatos lecheros con problemas de papilomatosis bovina (Vindas et al., 2013).

Morfológicamente, el sarcoide equino es una neoplasia de tejido fibroso, de origen fibroblástico, no metastásico, que afecta principalmente la epidermis (Knottenbelt, 2005). Histológicamente, se caracteriza por la proliferación dérmica densa de fibroblastos, formando nidos y asas entrelazadas. Según Scott y Miller (2011), esta neoplasia se puede clasificar de acuerdo con su apariencia clínica en Tipo I: Verrugoso, de superficie seca y queratinizada, semejante a un papiloma o coliflor; Tipo II: Fibroblástico, de aspecto se- mejante a un granuloma; Tipo III: Mixto, como combinación de los dos anteriores; y Tipo IV: una forma oculta de sarcoide, de piel ligeramente engrosada y superficie alopécica.

El tratamiento del sarcoide equino depende del tipo y localización. Se han propuesto algunas alternativas como la cirugía, criocirugía y crioterapia, así como la quimioterapia tópica e intralesional, radioterapia e inmunoterapia (Souza et al., 2007). Existen reportes de reducción de las lesiones tumorales con el uso intratumoral de 5fluorouracil con $61 \%$ de recuperación (Stewart et al., 2006). Por lo anteriormente señalado, el objetivo del presente artículo fue presentar la descripción de un caso clínico de sarcoide equino de tipo fibroblástico, su manejo clínico, hallazgos histológicos y resolución quirúrgica.

\section{Caso Clínico}

\section{Descripción del Caso}

Se presenta el caso de un paciente equino de 6 años, macho entero, de raza Paso Fino Colombiano, con un peso de $350 \mathrm{~kg}$, proveniente de una finca ubicada en el municipio Sabana de Torres, departamento Santander, Colombia. El propietario manifestó que el paciente mostraba la presencia de un aumento de volumen o masa en la región del pene y prepucio (Figura 1A) desde hacía ocho meses aproximadamente. También señaló que el animal manifestaba dificultad para orinar, e incluso a veces mostraba incomodidad para caminar. Al paciente se le había removido quirúrgicamente un tumor prepucial de $3 \times 2 \mathrm{~cm}$ un año antes, sin que se le hubiera hecho el examen histopatológico.

El paciente se mostraba algo deprimido al examen físico, pero con excelente condición corporal. Presentaba buen tiempo de llenado capilar, mucosas oculares normales, temperatura corporal (TC) de $39.2^{\circ} \mathrm{C}$, frecuencia respiratoria (FR) de 30 respiraciones/min 
y frecuencia cardíaca (FC) de 95 latidos/min. El paciente tenía pulso arterial fuerte (arteria maxilar externa). Los linfonódulos superficiales sin alteraciones patológicas.

A la inspección de la región prepucial se observó una gran masa de aspecto irregular, hemorrágica (Figura 1B) y muy sensible. Se solicitó hematología completa y se sugirió la remoción quirúrgica del tumor y estudio histopatológico. De acuerdo con el examen clínico se propusieron como diagnósticos presuntivos habronemosis cutánea, sarcoide equino, papilomavirus y sarcoma equino.

\section{Preparación para la Cirugía}

Recomendaciones prequirúrgicas. El paciente fue sometido a un ayuno de 12 horas previo a la cirugía y se indicó el siguiente protocolo terapéutico: Toxoide anti-tetánico (1 $\mathrm{ml}$ vía subcutánea dos semanas previas a la cirugía), fenibutazona al $20 \%$ en solución inyectable ( $15 \mathrm{ml}$ vía endovenosa lentamente los tres días previos a la cirugía), Pencivet (penicilina $\mathrm{G}$ sódica, potásica y benzatínica) más estreptomicina y diclofenaco sódico (6x10 6 U [15 ml] vía intramuscular, 72 y 96 horas previas a la cirugía).

Protocolo preanestésico. Se empleó el maleato de acepromazina al $1 \%$, dosis de 0.05 $\mathrm{mg}$ por $\mathrm{kg} \mathrm{PV}(1 \mathrm{ml} / 100 \mathrm{~kg} \mathrm{PV})$, vía endovenosa.

Protocolo anestésico (anestesia disociativa). Se empleó una solución de clorhidrato de ketamina al 5\% $(50 \mathrm{mg} / \mathrm{ml}$ de clorhidrato de ketamina). Se administró una dosis de inducción de $2 \mathrm{mg}$ por $\mathrm{kg}$ PV por vía endovenosa. Luego, se suministraron bolos de mantenimiento de $1 \mathrm{mg}$ de clorhidrato de ketamina por cada kilogramo de peso vivo.

\section{Remoción Quirúrgica}

La cirugía se realizó en condiciones de campo debido a la dificultad de trasladar al paciente a un centro de salud veterinario. Se tomaron las condiciones mínimas de asepsia para evitar posibles contaminaciones. Se realizó exposición del pene (por acción de la acepromazina) (Figura 2a), y el área quirúrgica de lavó con solución yodada al 1\%. Todo el material de descarte e instrumental quirúrgico fue esterilizado en seco.

El paciente fue anestesiado y derribado. Básicamente, la extirpación se realizó mediante divulsión del tejido neoplásico hasta el mucocutáneo (Figuras 2b,c,d), de manera de extirpar la totalidad de la masa tumoral (Figura 3). Igualmente se extirpó una masa tumoral que se encontraba en la corona del glande. Se colectó un trozo de tejido $(1 \mathrm{x} 1 \mathrm{~cm})$ en formol al $10 \%$ para el estudio histológico. Además, se tomaron $80 \mathrm{~g}$ del mismo tejido para preparar una vacuna experimental. Esta muestra fue transportada al laboratorio en condiciones de refrigeración.

\section{Análisis de Laboratorio}

En el Cuadro 1 se muestran los resultados del análisis hematológico. Se observa un aumento de la serie blanca (leucocitosis) con neutrofilia. La serie roja y los trombocitos se

Cuadro 1. Valores hematológicos y contaje de plaquetas en el equino con sarcoma equino

\begin{tabular}{lcc}
\hline Prueba & Resultado & $\begin{array}{c}\text { Valor } \\
\text { normal* }^{*}\end{array}$ \\
\hline $\mathrm{Hm}(\%)$ & 45 & $38-53$ \\
$\mathrm{Hb}(\mathrm{g} \%)$ & 15 & $12-18$ \\
Gl. rojos (x10 $)$ & 6.8 & $4.5-7.1$ \\
$\mathrm{VCM}(\mathrm{fl})$ & 63 & $63-71$ \\
$\mathrm{HCM}(\mathrm{pb})$ & 21 & $20-23$ \\
$\mathrm{CHCM}(\mathrm{g} / \mathrm{dl})$ & 31 & $30-35$ \\
Gl. blancos (mlx10 $)$ & 15 & $7.8-12.5$ \\
Neutrófilos (\%) & 78 & 76 \\
Linfocitos (\%) & 20 & 20 \\
Eosinófilos (\%) & 1 & 1 \\
Monocitos (\%) & 1 & 3 \\
Plaquetas (mlx10 $\left.10^{3}\right)$ & 340 & $180-450$ \\
\hline
\end{tabular}

* Fuente: Arias et al. (2006) 


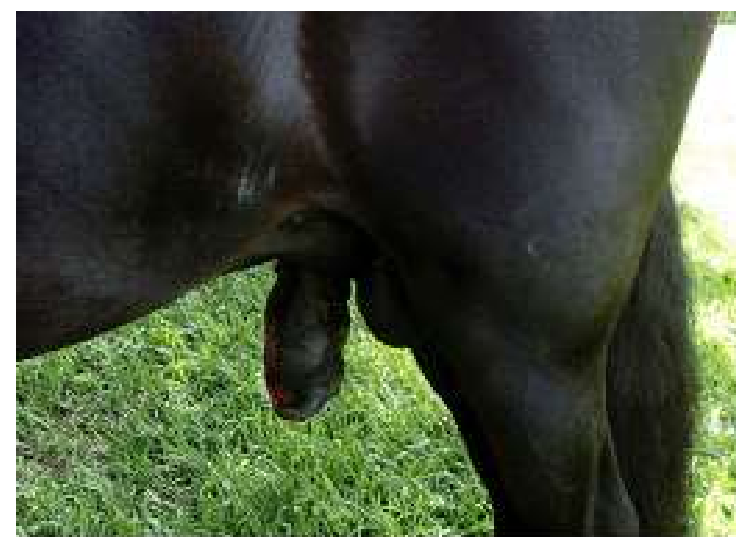

(A)

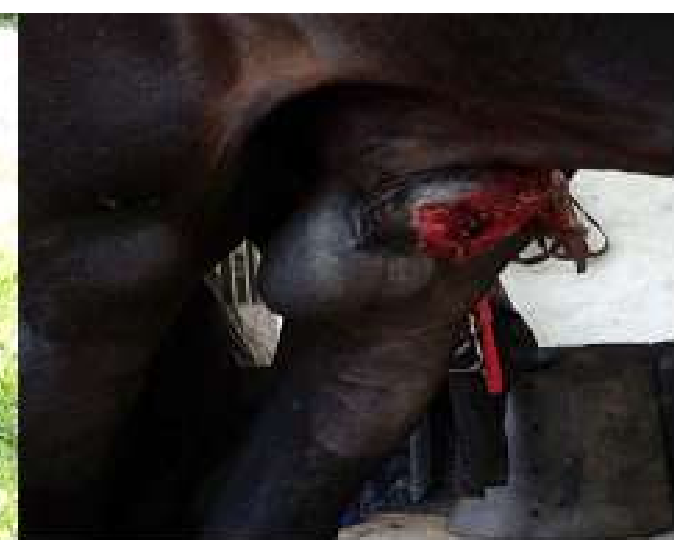

(B)

Figura 1. Paciente equino de 6 años, Paso Fino Colombiano. (A) Masa de aspecto irregular y hemorrágica en región prepucial y pene. (B) Aumento de volumen del pene

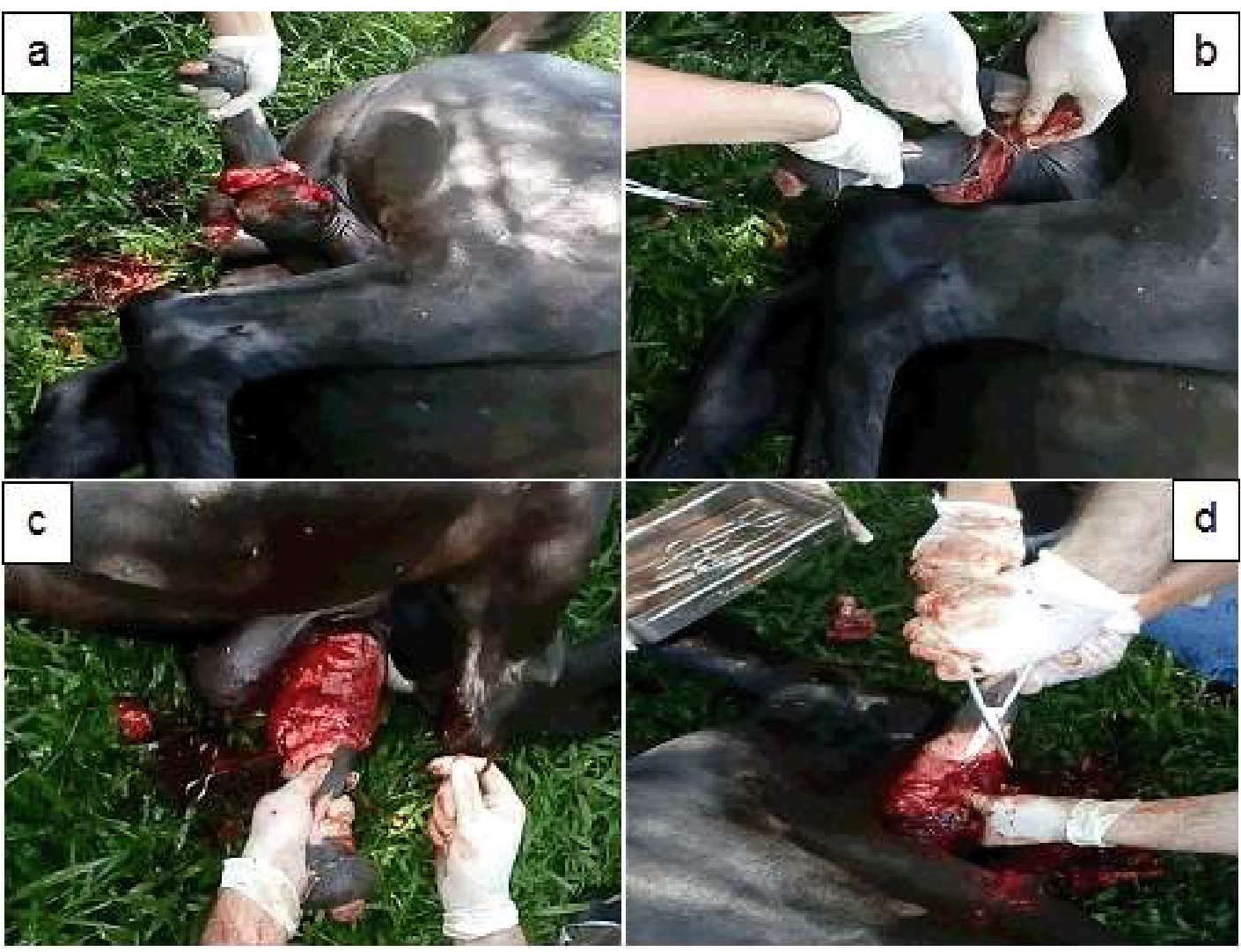

Figura 2. Paciente equino de 6 años, Paso Fino Colombiano. Cirugía para remoción de sarcoma equino. (a) exposición del pene, (b, c, d) divulsión y escisión de tejido neoplásico 
encontraban dentro de sus valores normales.

Los resultados hemáticos obtenidos señalan la presencia de un proceso infeccioso bacteriano de evolución aguda, secundario a la lesión tumoral. A la inspección, el tumor mostraba zonas con material purulento, que posiblemente procedía de focos de contaminación del medio ambiente.

Con respecto al estudio microscópico, la Figura 4 muestra un corte histológico del tejido tumoral coloreado con hematoxilinaeosina. Específicamente, la Figura 4A muestra una hiperqueratosis de la epidermis con proyecciones sobre la lámina basal de la dermis, mientras que en la Figura 4B se observa una reacción fibrosa multifocal con áreas de respuesta inflamatoria focal extensiva por debajo de la membrana basal de la epidermis. La Figura 5 muestra un corte histológico con diversos grados de reacción inflamatoria, infiltrados de células fibroblásticas y polimorfonucleares. En la figura $5 \mathrm{~A}$ se observan tres focos de necrosis de licuefacción, detritus celulares y reacción fibrosa, con predominio de fibras de tejido conectivo en fondo del campo coloración azul y patrón inflamatorio (flecha). En la figura 5B se muestra una reacción inflamatoria mixta con predominio de polimorfonucleares (neutrófilos), con fragmentación de las fibras de colágeno $\mathrm{y}$ focos de necrosis de licuefacción. El resultado del diagnóstico histológico fue una reacción de tipo piogranulomatosa, compatible con sarcoide equino.

Con respecto al diagnóstico diferencial, la habronemosis cutánea es un tipo de miasis ocasionada por la mosca Habronema sp; sin embargo, la ausencia de larvas en las masas ulceradas descarta la presencia de este parásito. En cuanto a un papilomavirus, se descarta su presencia, ya que generalmente estos tienen resolución espontánea. Por otro lado, el estudio histológico descarta la posibilidad de un sarcoma, debido a la ausencia de células indiferenciadas y presencia de células en proceso de mitosis.
Las características clínico-patológicas del tumor extirpado, así como del estudio histológico confirman el diagnóstico de sarcoide equino de tipo fibroblástico. El aspecto tumoral y granulomatoso de la masa extirpada de la región peniana y prepucial coinciden con los hallazgos señalados por Cardona et al. (2013) en un burro de Montería. Igualmente, los resultados macroscópicos coinciden con lo descrito por otros autores (Knottenbelt, 2005; Thomassian, 2005; Cremasco y Sequera, 2010), quienes señalaron la apariencia granulomatosa, crecimiento exagerado e invasivo, consistencia firme, fibrosa y difusamente ulcerado, como signos típicos del sarcoide equino. Igualmente, los hallazgos histopatológicos coinciden con Cardona et al. (2013), quienes señalaron hiperplasia epidérmica, hiperqueratosis, largas proyecciones epiteliales en dirección a la dermis y orientación perpendicular de los fibroblastos con relación a la membrana basal.

La edad del equino coincide con otros informes que reportaron esta patología en equinos entre 3 y 6 años (Bensignor et al., 2005; Ginn et al., 2007; Santos et al., 2011).

\section{Tratamiento Posoperatorio}

Se aplicó fenilbutazona al $20 \%$ en solución inyectable ( $15 \mathrm{ml})$, vía endovenosa, por dos días; pencivet $\left(6 \times 10^{3} \mathrm{UI}\right)$, vía intramuscular el día de la cirugía y a las 48 horas; hisopados intraprepuciales con una solución de glicerina, lidocaína al 2\% y sábila, para evitar adherencias y posteriores complicaciones; y autohemoterapia, terapia que ha sido descrita para casos de papilomavirus en ganado vacuno (Hegde, 2011) en dosis única de $30,25,20$ y $15 \mathrm{ml}$, vía intramuscular, la semana $1,2,3$ y 4 , respectivamente.

\section{Agradecimientos}

El autor agradece al personal de la hacienda La Isabela por la colaboración prestada en la ejecución del presente trabajo de campo. Así mismo, a la Universidad de 


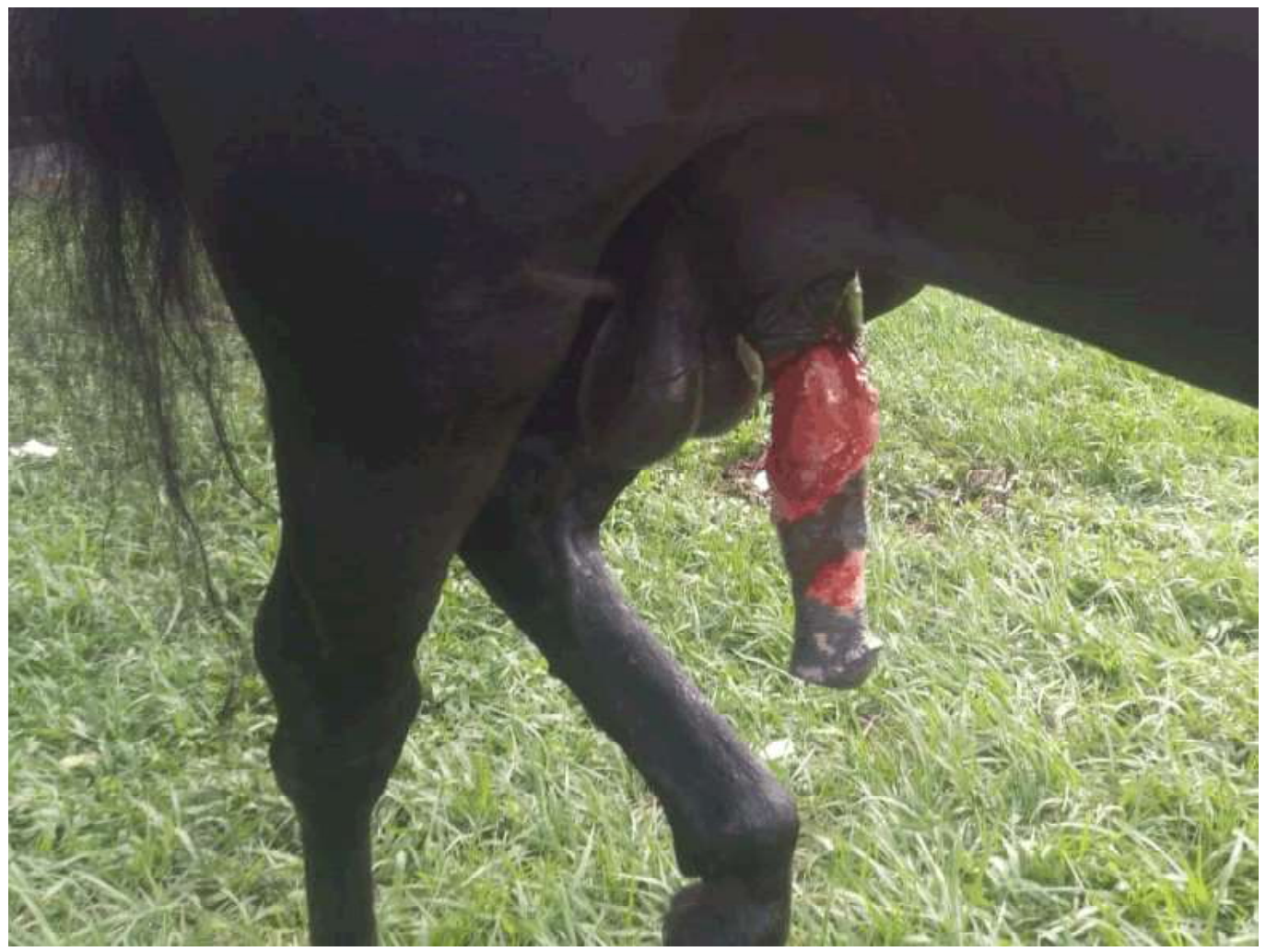

Figura 3. Paciente equino de 6 años, Paso Fino Colombiano. Vista del animal y del pene luego de la extirpación total del tejido neoplásico

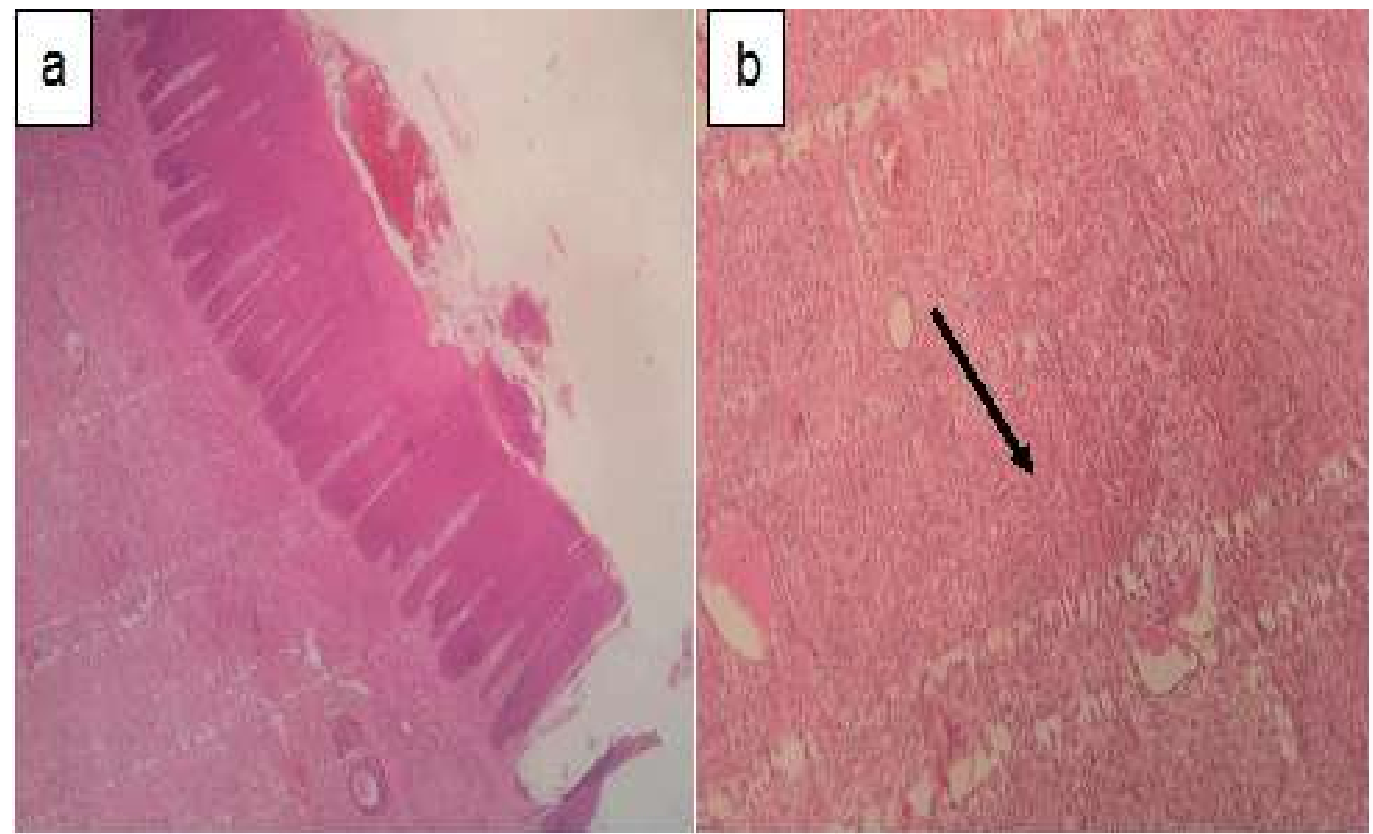

Figura 4. Histología del sarcoide equino: (a) Proyecciones del tejido epidermal. H-E, 100x. (b) Reacción inflamatoria (flecha). H-E. 40x 

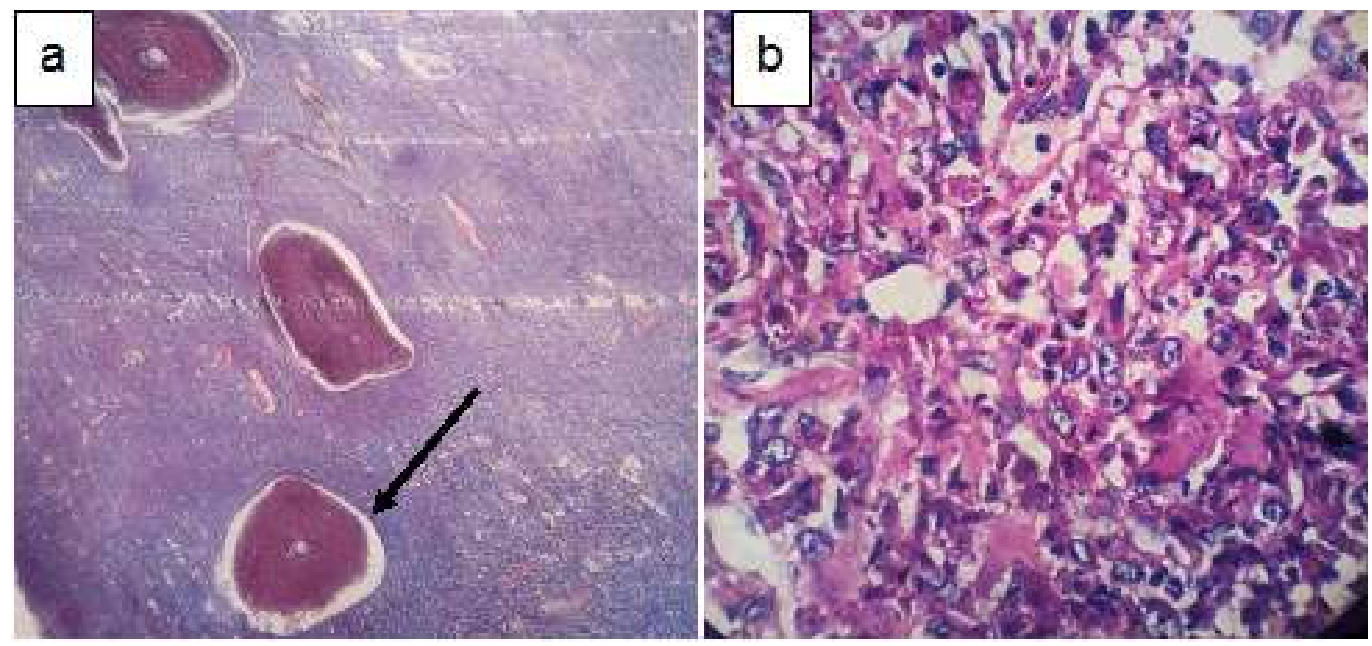

Figura 5. Histología del sarcoide equino: (a) Tres focos de necrosis de licuefacción (flecha). Tricrómico de Mason. 100x. (b) Reacción inflamatoria mixta. PAS, 1000x.

Santander por el apoyo prestado en la redacción del presente artículo.

\section{Literatura Citada}

1. Arias M, Naranjo M del P, Restrepo A. 2006. Comparación de los valores del hemoleucograma según la edady el sexo en caballos Pura Sangre Inglés del hipódromo Los Comuneros de Guarne, Antioquia. Revista CES MVZ 1: 14-21.

2. Bensignor E, Groux D, Lebis C. 2005. As doenças de pele no cavalo. São Paulo: Ed Andrei. 128 p.

3. Bergvall KE. 2013. Sarcoids. Vet Clin NAm-Equine 29: 657-671. doi: 10.1016/ j.cveq.2013.09.002

4. Cardona J, Vargas M, Perdomo S. 2013. Sarcoide equino fibroblástico periocular en un burro (Eqqus asinus). Ces Med Vet Zootec 8: 97-106.

5. Cremasco A, Sequera J. 2010. Sarcoide equino. Aspectos clínicos, etiológicos y anatomopatológicos. Vet Zootec 17: 191-199.
6. Finlay $M$, Yuan $Z$, Burden $F$, Trawford A, Morgan I, Campo M, Nasir L. 2009. The detection of bovine papillomavirus type 1 DNA in flies. Virus Res 144: 315-317. doi: 10.1016/ j.virusres.2009.04.015

7. Ginn P, Mansell J, Rakich P. 2007. Skin and appendages. In: Pathology of domestic animals. $5^{\text {th }}$ ed. Philadelphia: Ed Elsevier. p 553-781.

8. Hegde G. 2011. Cutaneous papillomatosis in a non-descript cow. Vet Sci Res J 2: 37- 38.

9. Jubb KV, Kennedy PC, Palmer N. 2007. Pathology of domestic animals. $5^{\text {th }}$ ed. USA: Elsevier Saunders, USA. 2340 p.

10. Knottenbelt DC, Mattews JB. 2001. A positive step forwards in the diagnosis of equine sarcoid. Vet J 161: 224- 226. doi: $10.1053 /$ tvj1.2000.0555

11. Knottenbelt DA. 2005. Suggested clinical classification for the equine sarcoid. Clin Tech Equine Pract 4: 278-295

12. McMahlan NJ, Dubovi EJ. 2011. Fenner's veterinary virology. $4^{\text {th }} \mathrm{ed}$. USA: Academic Press. 534 p. 
13. Santos D, Avanza M, Dias L, Lot R. 2011. Sarcóide fibroblástico periocular em equino - Relato de caso. Rev Cient Elect Med Vet 9(16). [Internet]. Disponible en: http://faef.revista.inf.br/ imagens_arquivos/arquivos_destaque/ Zgutf8Tiro0cudt 2013-6-25-17-4419.pdf

14. Scott WD, Miller HW. 2003. Neoplastic and nonneoplastic tumors. In: Equine dermatology. Saint Louis, USA: Ed Saunders. p 698-795.

15. Scott WD, Miller HW. 2011. Equine dermatology. $2^{\text {nd }}$ ed. USA: Saunders. $736 \mathrm{p}$.

16. Souza W, Fagundes E, Rocha E, Zangirolani D, Sacco S, Pereira D, Rosa E. 2007. Sarcóide equino. Relato de caso. Rev Cient Elect Med Vet 9(8). [Internet]. Disponible en: http:// faef.revista.inf.br/imagens_arquivos/
arquivos_destaque/Tpsr7eHCopMRqi1_2013-5-21-16-48-0.pdf

17. Sperotto J. 2010. Sarcoide equino. Tesis de Maestría. Santa María, Brasil: Univ. Federal de Santa María. $44 \mathrm{p}$.

18. Stewart AA, Rush B, Davis E. 2006. The efficacy of intratumoural 5fluorouracil for the treatment of equine sarcoids. Aust Vet J 84: 101-106.

19. Thomassian A. 2005. Enfermidades dos cavalos. $4^{\mathrm{a}}$ ed. São Paulo: Ed. Varela. 573 p.

20. Vindas $R$, Villagra $R$, Vargas $J$, Jiménez C, Marín R, Rodríguez $H$, Estrada M. 2013. Tratamiento de sarcoide equino: reporte de cuatro casos clínicos. Rev Cien Vet 31: 59-68.

21. Yuan Z, Gallagher A, Gault E, Campo M, Nasir L. 2007. Bovine papillomavirus infection in equine sarcoids and in bovine bladder cancers. Vet J 174: 599-604. doi: 10.1016/j.tvj1.2006.10.012 